\title{
Permanent prostate brachytherapy extracapsular radiation dose distributions: analysis of a multi- institutional database
}

\author{
Gregory S. Merrick, MD', Wayne M. Butler, PhD', Peter Grimm, DO², Mallory Morris, BS', Jonathan H. Lief, PhD', \\ Abbey Bennett, BS', Ryan Fiano, BS, MPH' \\ ISchiffler Cancer Center \& Wheeling Jesuit University. Wheeling WV, 2Prostate Cancer Center of Seattle, Seattle, Washington, USA
}

\begin{abstract}
Purpose: Periprostatic brachytherapy doses impact biochemical control. In this study, we evaluate extracapsular volumetric dosimetry following permanent prostate brachytherapy in patients entered in a multi-institutional community database.

Material and methods: In the database, 4547 patients underwent brachytherapy (3094 - ${ }^{125} \mathrm{I}, 1437$ - ${ }^{103} \mathrm{Pd}$ and 16 ${ }^{131} \mathrm{Cs}$ ). Using the originally determined prostate volume, a $5 \mathrm{~mm}$, 3-dimensional peri-prostatic anulus was constructed around the prostate (except for a $2 \mathrm{~mm}$ posterior margin), and evaluated in its entirety and in $90^{\circ}$ segments. Prostate dosimetric parameters consisted of a $V_{100}$ and $D_{90}$ while the annular dosimetry was reported as a $V_{100}$.

Results: The intraprostatic $\mathrm{V}_{100}$ and $\mathrm{D}_{90}$ for ${ }^{103} \mathrm{Pd}$, and ${ }^{125} \mathrm{I}$ were statistically comparable when stratified by isotope and/or monotherapy vs. boost. The overall mean $\mathrm{V}_{100}$ for the periprostatic annulus was $62.8 \%$. The mean $\mathrm{V}_{100}$ at the base $(51.6 \%)$ was substantially less than the apex $(73.5 \%)$ and midgland $(65.9 \%)$. In addition, for all patients, the anterior $\mathrm{V}_{100}$ $(45.7 \%)$ was less than the lateral $(68.8 \%)$ and the posterior $(75.0 \%)$. The geometric $\mathrm{V}_{100}$ annular differences were consistent when evaluated by isotope. Overall, the $\mathrm{V}_{100}$ was higher in the ${ }^{125} \mathrm{I}$ cohort.

Conclusions: The optimal extracapsular brachytherapy dose and radial extent remains unknown, but will prove increasingly important with reductions and/or elimination of supplemental external beam radiation therapy. The large multi-institutional community database demonstrates periprostatic annular doses that are not as robust as those in selected high volume brachytherapy centers, and may be inadequate for optimal biochemical control following monotherapeutic brachytherapy, especially in higher risk patients.
\end{abstract}

Key words: brachytherapy, dosimetry, prostate cancer, treatment margins.

\section{Purpose}

Permanent prostate brachytherapy represents a highly efficacious treatment for clinically localized prostate cancer with a defined relationship between dosimetric quality, biochemical outcome and complications [1,2]. Previous studies have demonstrated that long-term cancer control is related to intra-prostatic radiation dose and periprostatic treatment margins [3-6]. However, treatment margins can vary markedly between patients with comparable intra-prostatic dose distributions [5]. Because brachytherapy dose decreases by as much as 20 Gy per millimeter at the periphery of the target volume, as many as $50 \%$ of patients with a pre-treatment prostate specific antigen (PSA) $<10 \mathrm{ng} / \mathrm{mL}$ manifest extracapsular extension (EPE), and the radial extent of EPE is usually in the range of 2-5 mm [7-9], periprostatic treatment margins accomplished by either monotherapeutic brachytherapy and/or the addition of supplemental external beam radiation therapy (XRT) are necessary to ensure geographic coverage of potential sites of EPE.

Currently, there is interest in minimizing and/or eliminating supplemental XRT in patients with higher risk disease [10]. A reduction/elimination of supplemental XRT will mandate adequate brachytherapy periprostatic treatment margins to address possible EPE and to maximize long term cancer control. At the present time, there is no consensus regarding the extent of periprostatic margins or the dose necessary to sterilize EPE, even among brachytherapy experts [11]. Previously in the Pro-Qura database (Pro-Qura, Seattle, WA, USA), we have documented substantial differences in overall intraprostatic sector dosimetric quality [12]. In the current Pro-Qura evaluation, we evaluate extracapsular dose distributions in patients implanted with permanent prostate brachytherapy. Hopefully, analysis of the Pro-Qura database and other multi-institutional studies will illustrate po- 
tential population-based inadequacies and help establish national standards of care.

\section{Material and methods}

From August 1999 to December 2008, 4547 post-implant computed tomography (CT) scans in the Pro-Qura database were available for analysis. The CT scans originated from 129 Pro-Qura participating brachytherapists. Patients implanted at the authors institutions are not part of the ProQura database. The original post-implant prostate dosimetry was reported in terms of a $V_{100}$ (the percentage of the prostate volume covered by the prescription dose) and $\mathrm{D}_{90}$ (the maximum dose covering $90 \%$ of the prostate volume). All implants were pre-planned. Post implant CT was performed at a median of 30 days following brachytherapy.

The Pro-Qura technique for post-implant dosimetric analysis has been described in detail [13]. In this study, using the Pro-Qura defined post-implant prostate volume, a $5 \mathrm{~mm}$, 3-dimensional periprostatic annulus was constructed around the prostate gland (except posteriorly where a $2 \mathrm{~mm}$ margin was used), and evaluated in its entirety and in separate sectors to include the anterior, posterior, inferior and right/left apical aspects. Dose to the periprostatic annulus was defined in terms of an annular $V_{100}$. Figure 1 is an il-

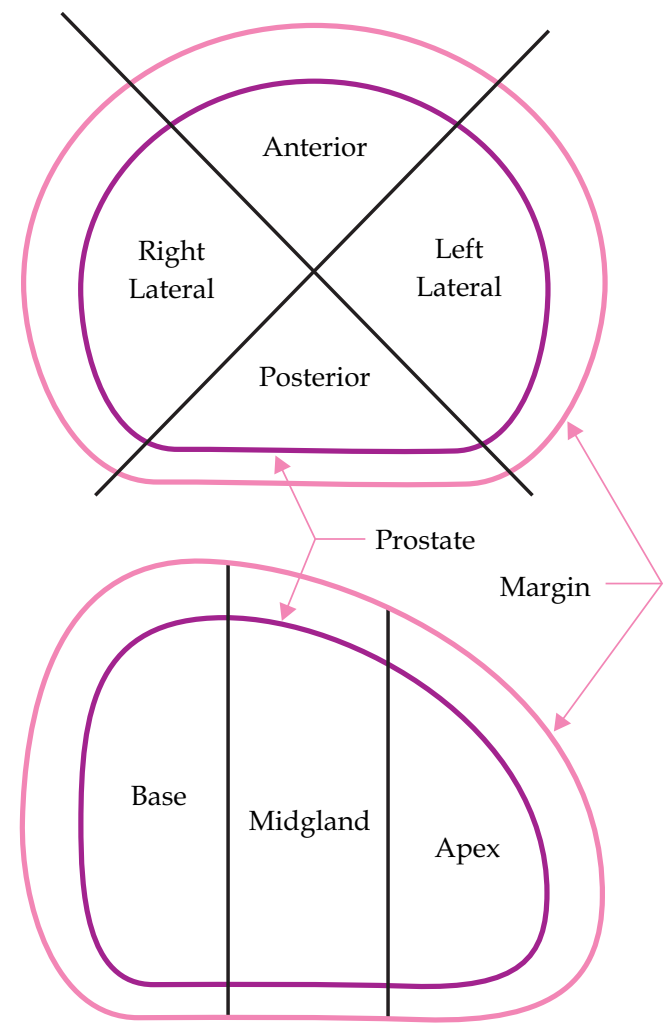

Fig. 1. Transverse (top) and sagittal (bottom) schematic of the prostate and its annular margin. The base, midgland, and apex were each divided into 4 transverse sectors producing 12 prostate and 12 annular sectors. The prostate was auto-margined by $5 \mathrm{~mm}$ in all directions except posterior, where the margin was $2 \mathrm{~mm}$ lustration of the periprostatic annulus and the individual segments.

Of the 4547 patients, 3094 (68.0\%) were implanted with ${ }^{125} \mathrm{I}, 1437(31.6 \%)$ with ${ }^{103} \mathrm{Pd}$ and $16(0.4 \%)$ with ${ }^{131} \mathrm{Cs}$. For ${ }^{125} \mathrm{I}, 84.3 \%$ of patients underwent monotherapy (144-145 Gy) and $15.7 \%$ a boost $(110 \mathrm{~Gy})$ for ${ }^{103} \mathrm{Pd}, 67.4 \%$ of patients underwent monotherapy (125 Gy) and $32.6 \%$ a boost ( $90-100 \mathrm{~Gy}$ ). For ${ }^{131} \mathrm{Cs}, 62.5 \%$ of patients underwent monotherapy (115 Gy) and $37.5 \%$ a boost ( $84 \mathrm{~Gy}$ ). Because of small patient numbers, ${ }^{131} \mathrm{Cs}$ patients were not included in the analysis of periprostatic treatment margins, but were included in Table 1 for completeness.

Statistical analysis was performed using Predictive Analytics Software (PASW), Statistics Version 17.0 (SPSS Inc., Chicago, IL, USA). The means for continuous variables were compared using independent-samples $t$-tests, and oneway analysis of variance and chi-square tests were used to compare distributions within categorical variables. Probabilities of deviation from the null hypothesis of no significant differences were marked if statistically significant, $p<5 \%$.

\section{Results}

Treatment and summary dosimetric data for the 4547 patients in the study population are summarized in Table 1. There was no statistically significant difference in prostate $V_{100}$ or $D_{90}$ when stratified by isotope or monotherapy vs. boost. In addition, there was no difference in prostate size or number of implanted seeds when stratified by isotope. However, for both ${ }^{125} \mathrm{I}$ and ${ }^{103} \mathrm{Pd}$, prostate glands were statistically larger and more seeds were implanted in the monotherapy vs. the boost cohorts.

Table 2 summarizes the mean margin sector analysis for $\mathrm{V}_{100}$ for the 4547 evaluated patients. For all sectors, the mean $\mathrm{V}_{100}$ was $62.8 \%$. The $\mathrm{V}_{100}$ at the base $(51.6 \%)$ was less than the apex $(73.5 \%)$ and the midgland (65.9\%). In addition, for the group as a whole, the anterior $\mathrm{V}_{100}(45.7 \%)$ was less than the lateral $(68.8 \%)$ and the posterior $(75.0 \%) \mathrm{V}_{100}$. Tables 3 and 4 describe the mean margin sector volumes, and $V_{100}$ for ${ }^{125} \mathrm{I}$ and ${ }^{103} \mathrm{Pd}$. The $\mathrm{V}_{100}$ for ${ }^{125} \mathrm{I}$ was greater than the $\mathrm{V}_{100}$ for ${ }^{103} \mathrm{Pd}$ both overall, and when evaluated by apex/ midgland/base and anterior/lateral/posterior sectors.

\section{Discussion}

Despite favorable long term biochemical control rates in patients treated with permanent prostate brachytherapy, the definition of a technically adequate implant including periprostatic dose distributions remains somewhat unclear $[14,15]$. However, data suggests that permanent cancer control is related to intraprostatic radiation dose and periprostatic treatment margins [3-6].

Since extracapsular treatment margins can vary substantially in patients with high quality intraprostatic brachytherapy [5], analysis of annular brachytherapy doses will become mandatory as supplemental XRT is phased out of higher risk brachytherapy protocols. Periprostatic doses are attainable via either a brachytherapy approach that includes generous periprostatic treatment margins and/or the addition of XRT $[3,4,11]$. 
Table 1. Treatment and summary dosimetric data for the 4547 patients in the study population stratified by radionuclide and implant type

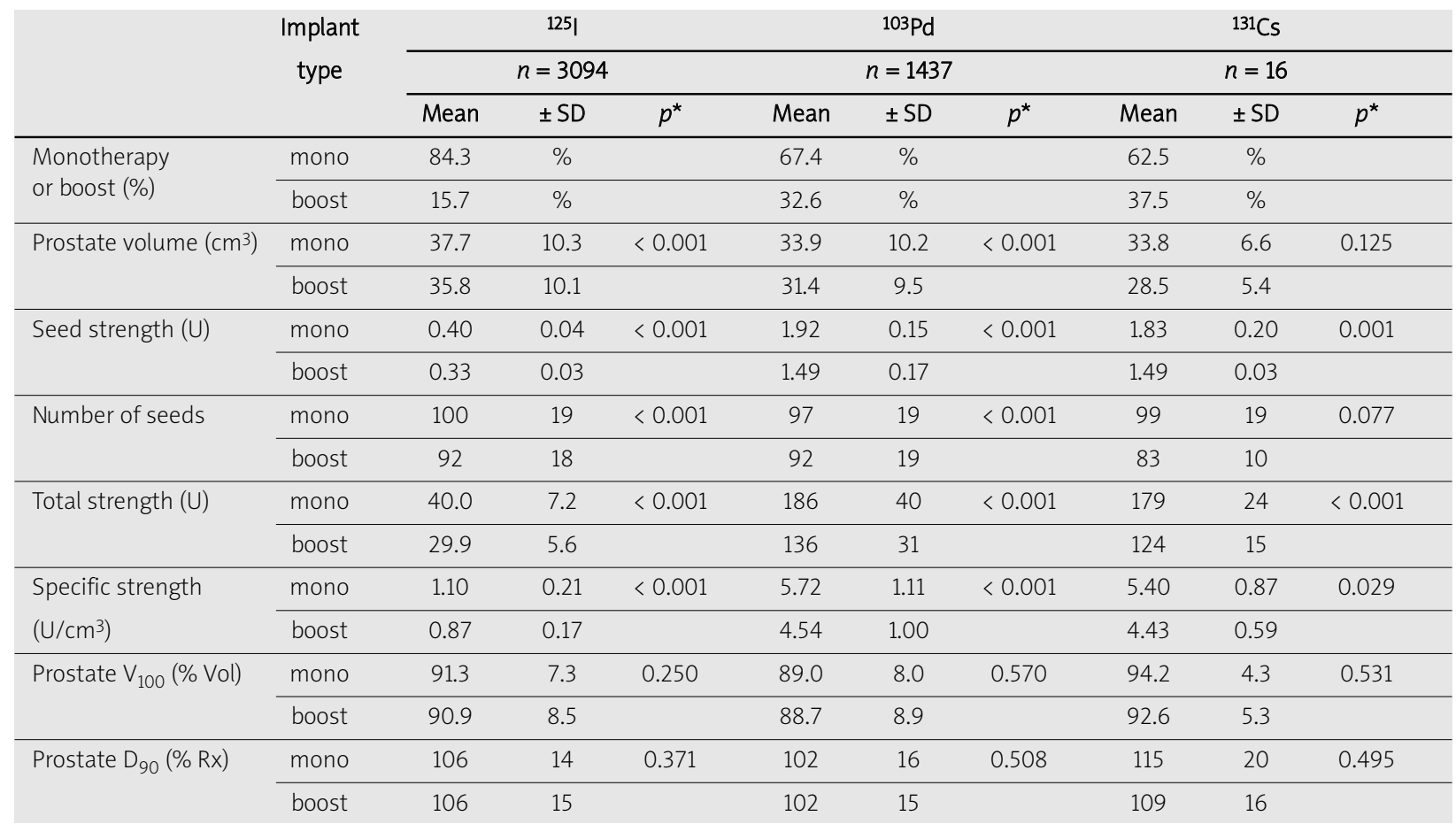

*Independent samples $t$-test. There was no significant difference between monotherapy or boost treatments for the percentage dosimetric parameters $V_{100}$ and $D_{90}$

Table 2. Mean margin sector volumes and $V_{100}$ for the 4531 patients analyzed

\begin{tabular}{lccccc} 
& Anterior & \multicolumn{2}{c}{ Left and right lateral } & Posterior & All \\
\hline Apex & Anterior apex & Left apex & Right apex & Posterior apex & All apex \\
\hline Volume $\left(\mathrm{cm}^{3}\right)$ & $1.69 \pm 0.6$ & $1.86 \pm 0.5$ & $1.84 \pm 0.45$ & $0.71 \pm 0.3$ & $6.09 \pm 1.4$ \\
\hline$V_{100}(\%)$ & $59.8 \pm 29$ & $78.1 \pm 22$ & $80.3 \pm 21$ & $77.8 \pm 25$ & $73.5 \pm 19$ \\
\hline Midgland & Anterior midgland & Left midgland & Right midgland & Posterior midgland & All midgland \\
\hline Volume $\left(\mathrm{cm}^{3}\right)$ & $2.36 \pm 0.6$ & $2.39 \pm 0.5$ & $2.39 \pm 0.5$ & $0.65 \pm 0.3$ & $7.80 \pm 1.6$ \\
\hline$V_{100}(\%)$ & $48.1 \pm 28$ & $70.7 \pm 24$ & $72.7 \pm 23$ & $90.0 \pm 18$ & $65.9 \pm 19$ \\
\hline Base & Anterior base & Left base & Right base & Posterior base & All base \\
\hline Volume $\left(\mathrm{cm}^{3}\right)$ & $2.18 \pm 0.6$ & $2.38 \pm 0.5$ & $2.38 \pm 0.5$ & $0.79 \pm 0.4$ & $7.73 \pm 1.8$ \\
\hline$V_{100}(\%)$ & $33.2 \pm 23$ & $57.7 \pm 24$ & $59.6 \pm 23$ & $62.7 \pm 28$ & $51.6 \pm 17$ \\
\hline All & All anterior & All lateral & & All posterior & All sectors \\
\hline Volume $\left(\mathrm{cm}^{3}\right)$ & $6.23 \pm 1.6$ & $13.2 \pm 2.7$ & & $2.15 \pm 0.7$ & $21.6 \pm 4.5$ \\
\hline$V_{100}(\%)$ & $45.7 \pm 21$ & $68.8 \pm 15$ & & $75.0 \pm 18$ & $62.8 \pm 14$
\end{tabular}

*Independent samples $t$-test. There was no significant difference between monotherapy or boost treatments for the percentage dosimetric parameters $V_{100}$ and $D_{90}$

Data suggests that periprostatic dose impacts the likelihood of treatment success $[5,6,9,16]$. Choi et al. demonstrated that the anterior treatment margin in low risk prostate cancer patients was statistically significant in predicting biochemical outcome [5]. In a more sophisticated study, Crook et al. using magnetic resonance imaging (MRI) defined prostate contours reported dosimetric coverage of the prostate gland with 2-, 3-, and 5-mm margins in patients biochemically controlled and in those with biopsy proven recurrence [16]. The mean $D_{90}$ and $V_{100}$ were statistically low- er in the 2-, 3-, and 5-mm expansions in patients with biopsy proven local failure. Among patients without and with local recurrence, the mean $\mathrm{V}_{100}$ in the 5 mm-group were $77.4 \%$ and $72.4 \%(p=0.045)$. Merrick et al. reported an annular $\mathrm{V}_{100}$ of $95.1 \%$ in a series of ${ }^{125} \mathrm{I}$ and ${ }^{103} \mathrm{Pd}$ patients [6]. In that study, margin status did not correlate with biochemical control, probably as a result of robust intraprostatic and annular dosimetry. In the current Pro-Qura study, the overall annular $\mathrm{V}_{100}$ was $62.8 \%$ for all evaluated patients (65.2\% for ${ }^{125} \mathrm{I}$ and $57.6 \%$ for ${ }^{103} \mathrm{Pd}$ ), which compared to the 
Table 3. Mean margin sector volumes and $V_{100}$ for the 3094125 Iodine patients analyzed

\begin{tabular}{|c|c|c|c|c|c|}
\hline & Anterior & Left and & t lateral & Posterior & All \\
\hline Apex & Anterior apex & Left apex & Right apex & Posterior apex & All apex \\
\hline Volume $\left(\mathrm{cm}^{3}\right)$ & $1.74 \pm 0.6$ & $1.90 \pm 0.5$ & $1.88 \pm 0.5$ & $0.72 \pm 0.3$ & $6.23 \pm 1.4$ \\
\hline $\mathrm{V}_{100}(\%)$ & $65.7 \pm 28$ & $81.6 \pm 20$ & $84.5 \pm 18$ & $81.0 \pm 23$ & $77.8 \pm 17$ \\
\hline Midgland & Anterior midgland & Left midgland & Right midgland & Posterior midgland & All midgland \\
\hline Volume $\left(\mathrm{cm}^{3}\right)$ & $2.43 \pm 0.6$ & $2.45 \pm 0.5$ & $2.45 \pm 0.5$ & $0.67 \pm 0.3$ & $8.00 \pm 1.6$ \\
\hline $\mathrm{V}_{100}(\%)$ & $52.0 \pm 28$ & $72.5 \pm 24$ & $75.2 \pm 22$ & $92.0 \pm 16$ & $68.6 \pm 20$ \\
\hline Base & Anterior base & Left base & Right base & Posterior base & All base \\
\hline Volume $\left(\mathrm{cm}^{3}\right)$ & $2.23 \pm 0.6$ & $2.44 \pm 0.5$ & $2.44 \pm 0.5$ & $0.82 \pm 0.4$ & $7.92 \pm 1.8$ \\
\hline $\mathrm{V}_{100}(\%)$ & $34.1 \pm 23$ & $57.8 \pm 24$ & $60.2 \pm 24$ & $63.76 \pm 28$ & $52.2 \pm 18$ \\
\hline All & All anterior & All lateral & & All posterior & All sectors \\
\hline Volume $\left(\mathrm{cm}^{3}\right)$ & $6.41 \pm 1.7$ & $13.6 \pm 2.7$ & & $2.20 \pm 0.8$ & $22.2 \pm 4.5$ \\
\hline $\mathrm{V}_{100}(\%)$ & $49.0 \pm 21$ & $70.8 \pm 15$ & & $77.1 \pm 17$ & $65.2 \pm 14$ \\
\hline
\end{tabular}

Table 4. Mean margin sector volumes and $V_{100}$ for the 1,437 103Palladium: patients analyzed

\begin{tabular}{lccccc} 
& Anterior & \multicolumn{2}{c}{ Left and right lateral } & Posterior & All \\
\hline Apex & Anterior apex & Left apex & Right apex & Posterior apex & All apex \\
\hline Volume $\left(\mathrm{cm}^{3}\right)$ & $1.57 \pm 0.5$ & $1.77 \pm 0.4$ & $1.74 \pm 0.4$ & $0.70 \pm 0.3$ & $5.78 \pm 1.3$ \\
\hline $\mathrm{V}_{100}(\%)$ & $47.2 \pm 29$ & $70.5 \pm 24$ & $70.9 \pm 23$ & $70.7 \pm 27$ & $64.1 \pm 20$ \\
\hline Midgland & Anterior midgland & Left midgland & Right midgland & Posterior midgland & All midgland \\
\hline Volume $\left(\mathrm{cm}^{3}\right)$ & $2.21 \pm 0.5$ & $2.27 \pm 0.5$ & $2.27 \pm 0.5$ & $0.61 \pm 0.2$ & $7.36 \pm 1.6$ \\
\hline$V_{100}(\%)$ & $39.7 \pm 26$ & $66.6 \pm 23$ & $67.0 \pm 23$ & $85.6 \pm 21$ & $60.0 \pm 18$ \\
\hline Base & Anterior base & Left base & Right base & Posterior base & All base \\
\hline Volume $\left(\mathrm{cm}^{3}\right)$ & $2.06 \pm 0.5$ & $2.26 \pm 0.5$ & $2.26 \pm 0.5$ & $0.73 \pm 0.3$ & $7.31 \pm 1.7$ \\
\hline$V_{100}(\%)$ & $31.5 \pm 22$ & $57.6 \pm 22$ & $58.4 \pm 22$ & $60.3 \pm 29$ & $50.4 \pm 16$ \\
\hline All & All anterior & All lateral & & All posterior & All sectors \\
\hline Volume $\left(\mathrm{cm}^{3}\right)$ & $5.85 \pm 1.3$ & $12.6 \pm 2.6$ & & $2.04 \pm 0.7$ & $20.5 \pm 4.3$ \\
\hline$V_{100}(\%)$ & $38.4 \pm 19$ & $64.4 \pm 15$ & & $70.5 \pm 19$ & $57.6 \pm 13$
\end{tabular}

above mentioned results from high volume brachytherapy centers are probably inadequate for monotherapeutic approaches, especially those with higher risk disease. Previously, it has been reported that ${ }^{125} \mathrm{I}$ resulted in higher annular doses compared to ${ }^{103} \mathrm{Pd}[6]$, but did not result in biochemical control differences.

Although the radiation dose needed to sterilize periprostatic disease is unknown, the dose to control extraprostatic disease is probably significantly less than the threshold intraprostatic doses, because the ratio of extraprostatic to intraprostatic cancer is in the range of $0.4 \%$ [17]. Eventually, predictive modeling and improved imaging may enable prostate brachytherapists to tailor treatment margins on a case by case basis $[18,19]$. Until these technologies become available, a 3-5 $\mathrm{mm}$ periprostatic treatment margin appears prudent $[6,9,16]$.

A strength of our analysis is the diverse representation of a large number of community brachytherapy practices with post-implant dosimetry performed with a consistent and highly reproducible technique. However, a limitation of the study is that once stored in the Pro-Qura database, the individual brachytherapist responsible for the CT scan is no longer identifiable and as such a learning curve analysis for margin assessment was not possible. Most importantly, there are limitations to the Pro-Qura post-implant dosimetric technique. Because the Pro-Qura technique uses the pre-implant TRUS determined prostate volume, it is highly probable that the actual annular doses are less than what is reported in this study. In addition, due to the rapid dose fall off at the periphery of the target volume, annular dose distributions are very sensitive to contouring errors. In the current study, this variable has been minimized [8]. Finally, ProQura was established as a preplanning and dosimetry service, and does not have access to outcomes including biochemical control and/or complications. This eliminates our ability to establish a dose response curve for the annular dose necessary to secure long-term biochemical control.

\section{Conclusions}

The optimal extracapsular brachytherapy dose and radial extent remains unknown, but will prove increasingly important with reductions and/or elimination of supple- 
mental external beam radiation therapy. The Pro-Qura database demonstrates periprostatic annular doses that are not as robust as those in selected high volume brachytherapy centers, and may be inadequate for optimal biochemical control following monotherapeutic brachytherapy, especially in higher risk patients.

\section{Conflict of interest}

Peter Grimm is an owner of Pro-Qura.

\section{References}

1. Taira AV, Merrick GS, Butler WM et al. Long-term outcome for clinically localized prostate cancer treated with permanent interstitial brachytherapy. Int J Radiat Oncol Biol Phys 2010; 79: 1336-1342.

2. Zelefsky MJ, Kuban DA, Levy LB et al. Multi-institutional analysis of long-term outcome for stages T1-T2 prostate cancer treated with permanent seed implantation. Int J Radiat Oncol Biol Phys 2007; 67: 327-333.

3. Merrick GS, Butler WM, Wallner KE et al. Dosimetry of an extracapsular anulus following permanent prostate brachytherapy. Am J Clin Oncol 2007; 30: 228-233.

4. Merrick GS, Butler WM, Wallner KE et al. Extracapsular radiation dose distribution after permanent prostate brachytherapy. Am J Clin Oncol 2003; 26: e178-e189.

5. Choi S, Wallner K, Merrick GS et al. Treatment margins predict biochemical outcomes after prostate brachytherapy. Cancer J 2004; 3: 175-180.

6. Bittner N, Merrick GS, Butler WM et al. The correlation between annular treatment margins and biochemical failure in prostate brachytherapy patients with optimized intraprostatic dosimetry. Brachytherapy 2011; 10: 409-415.

7. Partin AW, Mangold LA, Lamm DM et al. Contemporary update of prostate cancer staging nomograms (Partin Tables) for the new millennium. Urology 2001; 58: 843-848.

8. Dawson JE, Wu T, Roy T et al. Dose effects of seed placement deviations from preplanned positions in ultrasound guided prostate implants. Radiother Oncol 1994; 32: 268-270.

9. Davis BJ, Pisansky TM, Wilson TM et al. The radial distance of extraprostatic extension of prostate carcinoma: implications for prostate brachytherapy. Cancer 1999; 85: 2630-2637.

10. Merrick GS, Wallner KE, Butler WM et al. 20 Gy versus 44 Gy of supplemental external beam radiotherapy with palladium103 for patients with greater risk disease: results of a prospective randomized trial. Int J Radiat Oncol Biol Phys 2012; 82: e449e455.

11. Merrick GS, Butler WM, Wallner KE et al. Variability of prostate brachytherapy preimplant dosimetry: a multi-institutional analysis. Brachytherapy 2005; 4: 243-251.

12. Merrick GS, Butler WM, Grimm P et al. Multisector prostate dosimetric quality: analysis of a large community database. Brachytherapy 2014, in press. http://dx.doi.org/10.1016/ j.brachy.2013.08.003

13. Merrick GS, Grimm PD, Sylvester J et al. Initial analysis of ProQura: a multi-institutional database of prostate brachytherapy dosimetry. Brachytherapy 2007; 6: 9-15.

14. Aronowitz JN, Rivard MJ. The phylogeny of permanent prostate brachytherapy. J Contemp Brachytherapy 2013; 5: 89-92.

15. Knaup C, Mavroidis P, Stathakis S et al. Evaluation of the effect of the prostate volume change on tumor control probability in LDR brachytherapy. J Contemp Brachytherapy 2011; 3: 125-130.

16. Crook J, Patil N, Ma C et al. Magnetic resonance imagingdefined treatment margins in iodine- 125 prostate brachytherapy. Int J Radiat Oncol Biol Phys 2010; 77: 1079-1084.
17. Davis BJ, Haddock MG, Wilson TM et al. Treatment of extraprostatic cancer in clinically organ-confined prostate cancer by permanent interstitial brachytherapy: is extraprostatic seed placement necessary? Tech Urol 2000; 6: 70-77.

18. Ellis R, Zhou E, Fu P et al. Single photon emission computerized tomography with capromab pendetide plus computerized tomography image set co-registration independently predicts biochemical failure. J Urol 2008; 179: 1768-1773.

19. Augustin H, Fritz G, Ehammer T et al. Accuracy of 3-Tesla magnetic resonance imaging for the staging of prostate cancer in comparison to the Partin tables. Acta Radiol 2009; 50: 562-569. 